\title{
Noncommutative Sugawara construction
}

\author{
M. Ghasemkhani ${ }^{\mathrm{a}}$ \\ Department of Physics, Shahid Beheshti University, G.C., Evin, Tehran 19839, Iran
}

Received: 5 April 2015 / Accepted: 15 July 2015 / Published online: 28 July 2015

(C) The Author(s) 2015. This article is published with open access at Springerlink.com

\begin{abstract}
The noncommutative extension of the Sugawara construction for free massless fermionic fields in two dimensions is studied. We prove that the equivalence of the noncommutative Sugawara energy-momentum tensor and symmetric energy-momentum tensor persists in the noncommutative extension. Some relevant physical results of this equivalence are also discussed.
\end{abstract}

\section{Introduction}

One of the outstanding features of two-dimensional field theories is bosonization, where a free massless fermionic field can be written as a bosonic field. This property is rooted in the work of Jordan and Wigner [1] where it was shown that the fermionic creation and annihilation operators may be represented as the bosonic counterparts. On the other hand, the idea of describing the strong interaction process in terms of currents was proposed in [2-4]. In this approach, the dynamical variables are taken to be the currents and the canonical formalism is abandoned. In other words, each particle does not correspond to a field which satisfies the canonical commutation relation but Hilbert space is built upon current operators. Accordingly, it was shown that the energy-momentum tensor of these theories can be expressed as a quadratic function of the currents known as the Sugawara construction [4]. Later on, it was proved that the symmetric energy-momentum tensor of the two-dimensional free massless fermionic theory is exactly equivalent to the Sugawara energy-momentum tensor which is bilinear in the fermionic currents [5].

Indeed, this equivalence confirmed the result of [1], the equivalence of free massless fermions and bosons, in an elegant way. Then generalization of this famous equivalence to the curved space-time was performed in [6] where the bosonfermion correspondence was shown for a general metric in two dimensions.

a e-mail: m_ghasemkhani@sbu.ac.ir
Our purpose is to study whether this equivalence is satisfied for noncommutative space, where the nature of the spacetime changes at very short distances [7-9], which is not a trivial extension. The authors in [10] considered the noncommutative generalization of the Sugawara energy-momentum tensor and then used the Seiberg-Witten map. While in the present work, the correspondence between the noncommutative Sugawara construction and the symmetric energymomentum tensor for two-dimensional free fermionic theory is addressed, without employing the Seiberg-Witten map. Applying the techniques described in [5], we demonstrate that the noncommutative Sugawara energy-momentum tensor exactly leads to the symmetric energy-momentum tensor. An interesting physical consequence of this equivalence is the noncommutative bosonization, which exhibits the relationship between the fermionic and bosonic fields in noncommutative space, as will be discussed in the last section.

\section{Equivalence of the symmetric energy-momentum tensor and Sugawara energy-momentum tensor in noncommutative space}

The first part of this section includes the derivation of the symmetric energy-momentum tensor using a variation of the action with respect to a generic metric. In the second part, we extend the Sugawara construction to noncommutative space and demonstrate that it will be equivalent to the symmetric energy-momentum tensor.

\subsection{Symmetric energy-momentum tensor}

Let us start from the noncommutative version of the free massless fermionic Lagrangian density, which is obtained by replacing the ordinary product with the star-product,

$\mathcal{L}=\frac{i}{2}\left(\bar{\psi} \gamma^{\alpha} \star \partial_{\alpha} \psi-\partial_{\alpha} \bar{\psi} \gamma^{\alpha} \star \psi\right)$, 
where the star-product is defined as follows:

$$
\left.f(x) \star g(x) \equiv \exp \left(\frac{i \theta_{\alpha \beta}}{2} \frac{\partial}{\partial a_{\alpha}} \frac{\partial}{\partial b_{\beta}}\right) f(x+a) g(x+b)\right|_{a, b=0} ;
$$

here $\theta_{\mu \nu}$ is an antisymmetric constant matrix. As is usual in two-dimensional field theory, we choose to work in Euclidean signature. In the noncommutative version, this has the added virtue that the Euclidean theory does not have issues with unitarity. The noncommutative symmetric energy-momentum tensor $T_{\mu \nu}^{\star}$ is achieved by variation of the action $S$ with respect to a generic metric $g_{\mu \nu}$ and setting $g^{\mu \nu}=\delta^{\mu \nu}$ in the end [11]:

$T_{\mu \nu}^{\star}=\left.\frac{2}{\sqrt{g}} \frac{\delta S}{\delta g^{\mu \nu}(x)}\right|_{g^{\mu \nu}=\delta^{\mu \nu}}$,

where $g$ indicates the determinant of the metric with signature $(+,+)$. The variation of the action corresponding to the Lagrangian density (2.1) can be written as

$$
\begin{aligned}
\delta S= & \frac{i}{8} \int d^{2} y \sqrt{g} \star\left(\bar{\psi} \star \gamma_{\alpha} \delta g^{\alpha \beta} \star \partial_{\beta} \psi+\bar{\psi} \star \gamma_{\alpha} \delta g^{\beta \alpha}\right. \\
& \left.\times \star \partial_{\beta} \psi-\partial_{\alpha} \bar{\psi} \star \gamma_{\beta} \delta g^{\alpha \beta} \star \psi-\partial_{\alpha} \bar{\psi} \star \gamma_{\beta} \delta g^{\beta \alpha} \star \psi\right) \\
& +\frac{i}{2} \int d^{2} y(\delta \sqrt{g}) \star\left(\bar{\psi} \gamma^{\alpha} \star \partial_{\alpha} \psi-\partial_{\alpha} \bar{\psi} \gamma^{\alpha} \star \psi\right) .
\end{aligned}
$$

Using Eq. (2.3) and the cyclic property of the star-product under the integral, we have

$$
\begin{aligned}
T_{\mu \nu}^{\star}= & -\frac{i}{4}\left(\partial_{\nu} \psi_{\beta} \star \bar{\psi}_{\alpha}\left(\gamma_{\mu}\right)^{\alpha \beta}+\partial_{\mu} \psi_{\beta} \star \bar{\psi}_{\alpha}\left(\gamma_{\nu}\right)^{\alpha \beta}\right. \\
& \left.+\partial_{\mu} \bar{\psi}_{\alpha} \star \psi_{\beta}\left(\gamma_{\nu}\right)^{\alpha \beta}+\partial_{\nu} \bar{\psi}_{\alpha} \star \psi_{\beta}\left(\gamma_{\mu}\right)^{\alpha \beta}\right) \\
& -\frac{i}{2} \delta^{\mu \nu}\left(\bar{\psi}_{\alpha} \star \partial_{\lambda} \psi_{\beta}\left(\gamma^{\lambda}\right)^{\alpha \beta}-\partial_{\lambda} \bar{\psi}_{\alpha} \star \psi_{\beta}\left(\gamma^{\lambda}\right)^{\alpha \beta}\right) .
\end{aligned}
$$

Applying the equation of motion for free massless fermions, we find the energy-momentum tensor as

$$
\begin{aligned}
T_{\mu \nu}^{\star}= & -\frac{i}{4}\left(\partial_{\nu} \psi_{\beta} \star \bar{\psi}_{\alpha}\left(\gamma_{\mu}\right)^{\alpha \beta}+\partial_{\mu} \psi_{\beta} \star \bar{\psi}_{\alpha}\left(\gamma_{\nu}\right)^{\alpha \beta}\right. \\
& \left.+\partial_{\mu} \bar{\psi}_{\alpha} \star \psi_{\beta}\left(\gamma_{\nu}\right)^{\alpha \beta}+\partial_{\nu} \bar{\psi}_{\alpha} \star \psi_{\beta}\left(\gamma_{\mu}\right)^{\alpha \beta}\right),
\end{aligned}
$$

which is completely symmetric under $\mu \leftrightarrow \nu$.

\subsection{Sugawara energy-momentum tensor}

The equivalence of the Sugawara construction and the symmetric energy-momentum tensor in commutative space has been shown in [5] and is reviewed in Appendix A. In the present section, we construct the noncommutative version of the Sugawara energy-momentum tensor to demonstrate that it is precisely equivalent to (2.6).

The Lagrangian (2.1) is invariant under a global $U(1)$ transformation which yields two different Noether currents $[12,13]$,

$$
\begin{gathered}
\mathcal{J}_{\mu}(x)=: \bar{\psi}_{\alpha}(x) \star \psi_{\beta}(x):\left(\gamma_{\mu}\right)^{\alpha \beta}, \\
J_{\mu}(x)=: \psi_{\beta}(x) \star \bar{\psi}_{\alpha}(x):\left(\gamma_{\mu}\right)^{\alpha \beta},
\end{gathered}
$$

where : : denotes normal ordering. Now, we extend the commutative Sugawara construction to the noncommutative one as a bilinear function of $J_{\mu}(x)$ with inserting star-product instead of the ordinary product,

$$
\begin{aligned}
T_{\mu \nu}^{S^{\star}}= & \frac{1}{2 c}\left(J_{\mu}(x) \star J_{\nu}(x)+J_{\nu}(x) \star J_{\mu}(x)\right. \\
& \left.-\delta_{\mu \nu} J^{\lambda}(x) \star J_{\lambda}(x)\right),
\end{aligned}
$$

where $c$ is the Schwinger constant, which appears in the equal-time commutator of currents. Since the mass dimension of the energy-momentum tensor and of the currents in two dimensions is equal to two and one, respectively, the coefficient $c$ should be dimensionless, while in four dimensions, it is a dimensionful quantity with the dimension of a mass square. The detailed analysis of the current algebra in two dimensions shows that the value of $c$ in noncommutative case is the same as the commutative one, $c=\frac{1}{\pi}$ [14]. To prove that (2.8) is exactly equivalent to (2.6), we need to regularize the operator products in (2.8). To this end, we use the point-splitting technique [15] and replace $J_{\mu}(x) \star J_{v}(x)$ with

$$
\lim _{\epsilon \rightarrow 0}\left(J_{\mu}(x+\epsilon) \star J_{v}(x)-\left\langle J_{\mu}(x+\epsilon) \star J_{v}(x)\right\rangle\right),
$$

which leads to

$$
\begin{aligned}
T_{\mu \nu}^{s^{\star}}= & \frac{\pi}{2} \lim _{\epsilon \rightarrow 0}\left(J_{\mu}(x+\epsilon) \star J_{\nu}(x)+J_{\nu}(x+\epsilon) \star J_{\mu}(x)\right. \\
& -\delta_{\mu \nu} J^{\lambda}(x+\epsilon) \star J_{\lambda}(x)-\left\langle J_{\mu}(x+\epsilon) \star J_{\nu}(x)\right\rangle \\
& \left.-\left\langle J_{\nu}(x+\epsilon) \star J_{\mu}(x)\right\rangle+\delta_{\mu \nu}\left\langle J^{\lambda}(x+\epsilon) \star J_{\lambda}(x)\right\rangle\right) .
\end{aligned}
$$

To perform some algebraic manipulations on (2.10), we employ the star-product definition (2.2),

$$
\begin{aligned}
J_{\mu}(x+\epsilon)= & \mathcal{F}_{a b}: \psi_{\beta}(x+\epsilon+a) \\
& \times \bar{\psi}_{\alpha}(x+\epsilon+b):\left.\right|_{a, b=0}\left(\gamma_{\mu}\right)^{\alpha \beta},
\end{aligned}
$$

where $\mathcal{F}_{a b}$ is an abbreviated notation for the exponential operator appearing in (2.2). Accordingly, the first term of 
Eq. (2.10) can be written as

$$
\begin{aligned}
J_{\mu}(x+\epsilon) \star J_{v}(x)= & \mathcal{F}_{f g} \mathcal{F}_{a b} \mathcal{F}_{c d}: \psi_{\beta}(x+\epsilon+f+a) \\
& \times \bar{\psi}_{\alpha}(x+\epsilon+f+b):: \psi_{\sigma}(x+g+c) \\
& \times \bar{\psi}_{\rho}(x+g+d): \\
& \times\left.\left(\gamma_{\mu}\right)^{\alpha \beta}\left(\gamma_{\nu}\right)^{\rho \sigma}\right|_{f, g, a, b, c, d=0}
\end{aligned}
$$

Using Wick's theorem (2.12) changes into

$$
\begin{aligned}
J_{\mu}(x+\epsilon) \star J_{\nu}(x)= & \mathcal{F}_{f g} \mathcal{F}_{a b} \mathcal{F}_{c d}\left(: \psi_{\beta}(x+\epsilon+f+a)\right. \\
& \times \bar{\psi}_{\alpha}(x+\epsilon+f+b) \psi_{\sigma}(x+g+c) \\
& \times \bar{\psi}_{\rho}(x+g+d): \\
& -: \psi_{\sigma}(x+g+c)\left\langle\psi_{\beta}(x+\epsilon+f+a)\right. \\
& \left.\times \bar{\psi}_{\rho}(x+g+d)\right\rangle \bar{\psi}_{\alpha}(x+\epsilon+f+b): \\
& -: \psi_{\beta}(x+\epsilon+f+a)\left\langle\psi_{\sigma}(x+g+c)\right. \\
& \left.\times \bar{\psi}_{\alpha}(x+\epsilon+f+b)\right\rangle \bar{\psi}_{\rho}(x+g+d): \\
& -\left\langle\psi_{\beta}(x+\epsilon+f+a) \bar{\psi}_{\rho}(x+g+d)\right\rangle \\
& \left.\times\left\langle\psi_{\sigma}(x+g+c) \bar{\psi}_{\alpha}(x+\epsilon+f+b)\right\rangle\right) \\
& \times\left.\left(\gamma_{\mu}\right)^{\alpha \beta}\left(\gamma_{\nu}\right)^{\rho \sigma}\right|_{f, g, a, b, c, d=0} .
\end{aligned}
$$

Rewriting the other terms of (2.10) similar to (2.13) and substituting them again into (2.10), we obtain

$$
\begin{aligned}
T_{\mu \nu}^{s^{\star}}= & \frac{\pi}{2} \lim _{\epsilon \rightarrow 0}\left[\mathcal{Q}_{\mu \nu}(x, \epsilon)-\mathcal{R}_{\mu \nu}(x, \epsilon)\right. \\
& -\mathcal{R}_{\nu \mu}(x, \epsilon)-\mathcal{S}_{\nu \mu}(x,-\epsilon) \\
& \left.-\mathcal{S}_{\mu \nu}(x,-\epsilon)-\delta_{\mu \nu}\left[\mathcal{R}_{\lambda}^{\lambda}(x, \epsilon)+\mathcal{S}_{\lambda}^{\lambda}(x,-\epsilon)\right]\right],
\end{aligned}
$$

with

$$
\begin{aligned}
\mathcal{Q}_{\mu \nu}(x, \epsilon)= & \mathcal{F}_{f g} \mathcal{F}_{a b} \mathcal{F}_{c d}:\left(\psi_{\beta}(x+\epsilon+f+a)\right. \\
& \times \bar{\psi}_{\alpha}(x+\epsilon+f+b) \\
& \times \psi_{\sigma}(x+g+c) \bar{\psi}_{\rho}(x+g+d)\left(\gamma_{\mu}\right)^{\alpha \beta}\left(\gamma_{\nu}\right)^{\rho \sigma} \\
& +\psi_{\beta}(x+\epsilon+f+a) \bar{\psi}_{\alpha}(x+\epsilon+f+b) \\
& \times \psi_{\sigma}(x+g+c) \bar{\psi}_{\rho}(x+g+d)\left(\gamma_{\nu}\right)^{\alpha \beta}\left(\gamma_{\mu}\right)^{\rho \sigma} \\
& -\psi_{\beta}(x+\epsilon+f+a) \bar{\psi}_{\alpha}(x+\epsilon+f+b) \\
& \times \psi_{\sigma}(x+g+c) \bar{\psi}_{\rho}(x+g+d) \delta_{\mu \nu} \\
& \left.\times\left(\gamma_{\lambda}\right)^{\alpha \beta}\left(\gamma_{\lambda}\right)^{\rho \sigma}\right):\left.\right|_{f, g, a, b, c, d=0}, \\
\mathcal{R}_{\mu \nu}(x, \epsilon)= & \mathcal{F}_{f g} \mathcal{F}_{a b} \mathcal{F}_{c d}: \psi_{\sigma}(x+g+c) \\
& \times\left\langle\psi_{\beta}(x+\epsilon+f+a) \bar{\psi}_{\rho}(x+g+d)\right\rangle \\
& \times \bar{\psi}_{\alpha}(x+\epsilon+f+b): \\
& \times\left.\left(\gamma_{\mu}\right)^{\alpha \beta}\left(\gamma_{\nu}\right)^{\rho \sigma}\right|_{f, g, a, b, c, d=0},
\end{aligned}
$$

$$
\begin{aligned}
\mathcal{S}_{\nu \mu}(x,-\epsilon)= & \mathcal{F}_{f g} \mathcal{F}_{a b} \mathcal{F}_{c d}: \psi_{\beta}(x+\epsilon+f+a) \\
& \times\left\langle\psi_{\sigma}(x+g+c) \bar{\psi}_{\alpha}(x+\epsilon+f+b)\right\rangle \\
& \times \bar{\psi}_{\rho}(x+g+d): \\
& \times\left.\left(\gamma_{\mu}\right)^{\alpha \beta}\left(\gamma_{\nu}\right)^{\rho \sigma}\right|_{f, g, a, b, c, d=0} .
\end{aligned}
$$

The field ordering appearing in the vacuum expectation value of the relation (2.15) is not time ordering and is defined as ${ }^{1}$

$S^{(+)}(x-y)=\psi(x) \bar{\psi}(y)-: \psi(x) \bar{\psi}(y):=\langle\psi(x) \bar{\psi}(y)\rangle$.

In view of the above definition, the quantities $\mathcal{R}_{\mu \nu}(x, \epsilon)$ and $\mathcal{S}_{\nu \mu}(x,-\epsilon)$ can be represented as

$$
\begin{aligned}
\mathcal{R}_{\mu \nu}(x, \epsilon)= & \mathcal{F}_{f g} \mathcal{F}_{a b} \mathcal{F}_{c d}: \psi_{\sigma}(x+g+c) \\
& \times \bar{\psi}_{\alpha}(x+\epsilon+f+b): \\
& \times S_{\beta \rho}^{(+)}(\epsilon+f+a-g-d)\left(\gamma_{\mu}\right)^{\alpha \beta} \\
& \times\left.\left(\gamma_{\nu}\right)^{\rho \sigma}\right|_{f, g, a, b, c, d=0}, \\
\mathcal{S}_{\nu \mu}(x,-\epsilon)= & \mathcal{F}_{f g} \mathcal{F}_{a b} \mathcal{F}_{c d}: \\
& \times \psi_{\beta}(x+\epsilon+f+a) \bar{\psi}_{\rho}(x+g+d): \\
& \times S_{\sigma \alpha}^{(+)}(g+c-\epsilon-f-b)\left(\gamma_{\mu}\right)^{\alpha \beta} \\
& \times\left.\left(\gamma_{\nu}\right)^{\rho \sigma}\right|_{f, g, a, b, c, d=0} .
\end{aligned}
$$

Converting Eq. (2.17) to the star-product form, we obtain

$$
\begin{aligned}
& \mathcal{R}_{\mu \nu}(x, \epsilon)=-\left(\gamma_{\mu} S^{(+)}(\epsilon) \gamma_{\nu}\right)^{\alpha \beta}: \bar{\psi}_{\alpha}(x+\epsilon) \star \psi_{\beta}(x): \\
& \mathcal{S}_{\nu \mu}(x,-\epsilon)=\left(\gamma_{\nu} S^{(+)}(-\epsilon) \gamma_{\mu}\right)^{\alpha \beta}: \psi_{\beta}(x+\epsilon) \star \bar{\psi}_{\alpha}(x):,
\end{aligned}
$$

where

$$
S^{(+)}(\epsilon)=-\frac{i}{2 \pi} \frac{\epsilon \xi \gamma^{\xi}}{\epsilon^{2}}
$$

Note that the minus sign in $\mathcal{R}_{\mu \nu}(x, \epsilon)$ comes from the odd permutation of the fermionic fields.

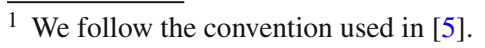


Expanding $\psi$ and $\bar{\psi}$ up to the first order in $\epsilon$ yields

$$
\begin{aligned}
\mathcal{R}_{\mu \nu}(x, \epsilon)= & \frac{i \epsilon^{\xi}}{2 \pi \epsilon^{2}}\left(\gamma_{\mu} \gamma_{\xi} \gamma_{\nu}\right)^{\alpha \beta}:\left[\bar{\psi}_{\alpha}(x)\right. \\
& \left.+\epsilon^{\eta} \partial_{\eta} \bar{\psi}_{\alpha}(x)+\mathcal{O}\left(\epsilon^{2}\right)\right] \star \psi_{\beta}(x):, \\
\mathcal{S}_{\nu \mu}(x,-\epsilon)= & \frac{i \epsilon^{\xi}}{2 \pi \epsilon^{2}}\left(\gamma_{\nu} \gamma_{\xi} \gamma_{\mu}\right)^{\alpha \beta}:\left[\psi_{\beta}(x)\right. \\
& \left.+\epsilon^{\eta} \partial_{\eta} \psi_{\beta}(x)+\mathcal{O}\left(\epsilon^{2}\right)\right] \star \bar{\psi}_{\alpha}(x):,
\end{aligned}
$$

and using the following symmetric limits:

$\lim _{\epsilon \rightarrow 0}\left(\frac{\epsilon^{\alpha}}{\epsilon^{2}}\right)=0, \quad \lim _{\epsilon \rightarrow 0}\left(\frac{\epsilon^{\alpha} \epsilon^{\beta}}{\epsilon^{2}}\right)=\frac{1}{2} \delta_{\alpha \beta}$,

we conclude that

$$
\begin{aligned}
& \lim _{\epsilon \rightarrow 0} \mathcal{R}_{\mu \nu}(x, \epsilon)=\frac{i}{4 \pi}\left(\gamma_{\mu} \gamma_{\xi} \gamma_{\nu}\right)^{\alpha \beta}: \partial^{\xi} \bar{\psi}_{\alpha}(x) \star \psi_{\beta}(x):, \\
& \lim _{\epsilon \rightarrow 0} \mathcal{S}_{\nu \mu}(x,-\epsilon)=\frac{i}{4 \pi}\left(\gamma_{\nu} \gamma_{\xi} \gamma_{\mu}\right)^{\alpha \beta}: \partial^{\xi} \psi_{\beta}(x) \star \bar{\psi}_{\alpha}(x): .
\end{aligned}
$$

Inserting the result (2.22) in (2.14), we arrive at

$$
\begin{aligned}
& T_{\mu \nu}^{s^{\star}}=\frac{\pi}{2} \mathcal{Q}_{\mu \nu}(x)-\frac{i}{8}:\left[\left(\gamma_{\mu} \gamma_{\xi} \gamma_{\nu}+\gamma_{\nu} \gamma_{\xi} \gamma_{\mu}\right)^{\alpha \beta}\right. \\
& \quad \times\left(\partial^{\xi} \bar{\psi}_{\alpha}(x) \star \psi_{\beta}(x)+\partial^{\xi} \psi_{\beta}(x) \star \bar{\psi}_{\alpha}(x)\right) \\
& \left.\quad+\delta_{\mu \nu}\left(\gamma^{\lambda} \gamma_{\xi} \gamma_{\lambda}\right)^{\alpha \beta}\left(\partial^{\xi} \bar{\psi}_{\alpha}(x) \star \psi_{\beta}(x)+\partial^{\xi} \psi_{\beta} \star \bar{\psi}_{\alpha}\right)\right]: .
\end{aligned}
$$

The product of gamma matrices in (2.23) can be simplified using the Clifford algebra,

$$
\begin{aligned}
& \gamma_{\mu} \gamma_{\xi} \gamma_{\nu}+\gamma_{\nu} \gamma_{\xi} \gamma_{\mu}=2\left(\delta_{\mu \xi} \gamma_{\nu}+\delta_{\nu \xi} \gamma_{\mu}-\delta_{\mu \nu} \gamma_{\xi}\right), \\
& \gamma_{\lambda} \gamma_{\xi} \gamma^{\lambda}=(2-d) \gamma_{\xi} .
\end{aligned}
$$

Substituting (2.24) into (2.23) and applying the equation of motion, $\gamma^{\xi} \partial_{\xi} \psi=0$ and $\partial_{\xi} \bar{\psi} \gamma^{\xi}=0$, we obtain the Sugawara energy-momentum tensor

$$
\begin{aligned}
T_{\mu \nu}^{s^{\star}}= & \frac{\pi}{2} \mathcal{Q}_{\mu \nu}(x)-\frac{i}{4}:\left[\left(\partial_{\nu} \bar{\psi}_{\alpha}(x) \star \psi_{\beta}(x)\right.\right. \\
& \left.+\partial_{\nu} \psi_{\beta}(x) \star \bar{\psi}_{\alpha}(x)\right)\left(\gamma_{\mu}\right)^{\alpha \beta} \\
& \left.+\left(\partial_{\mu} \bar{\psi}_{\alpha}(x) \star \psi_{\beta}(x)+\partial_{\mu} \psi_{\beta}(x) \star \bar{\psi}_{\alpha}(x)\right)\left(\gamma_{\nu}\right)^{\alpha \beta}\right]: .
\end{aligned}
$$

We notice that the last term of (2.23) vanishes in two dimensions as a result of the identity $\gamma_{\lambda} \gamma_{\xi} \gamma^{\lambda}=(2-d) \gamma_{\xi}$. In order to show that $T_{\mu \nu}^{\star}=T_{\mu \nu}^{s^{\star}}$, it is enough to demonstrate $\mathcal{Q}_{\mu \nu}=0$. For simplicity, we carry out computations in the light-cone coordinate system, $x_{ \pm}=x_{1} \pm i x_{2}$. The representation of the Euclidean gamma matrices,

$\gamma_{1}=\left(\begin{array}{cc}0 & -i \\ i & 0\end{array}\right), \quad \gamma_{2}=\left(\begin{array}{cc}0 & -1 \\ -1 & 0\end{array}\right)$,

in the light-cone coordinates is given by

$$
\begin{aligned}
& \gamma_{+}=\gamma_{1}+i \gamma_{2}=\left(\begin{array}{cc}
0 & -2 i \\
0 & 0
\end{array}\right), \\
& \gamma_{-}=\gamma_{1}-i \gamma_{2}=\left(\begin{array}{cc}
0 & 0 \\
2 i & 0
\end{array}\right),
\end{aligned}
$$

with

$g^{\mu \nu}=\left(\begin{array}{ll}g^{++} & g^{+-} \\ g^{-+} & g^{--}\end{array}\right)=\left(\begin{array}{cc}0 & \frac{1}{2} \\ \frac{1}{2} & 0\end{array}\right)$.

The equation of motion for two-dimensional massless fermions, which is described by $i \gamma^{\mu} \partial_{\mu} \psi=0$ with $\psi=$ $\left(\begin{array}{l}\psi_{1} \\ \psi_{2}\end{array}\right)$, in the light-cone coordinate system reduces to $\partial_{+} \psi_{1}=\partial_{-} \psi_{2}=0$. Thus

$$
\begin{gathered}
\psi_{1}=\psi_{1}\left(x_{-}\right), \quad \psi_{2}=\psi_{2}\left(x_{+}\right), \\
\bar{\psi}_{1}=\bar{\psi}_{1}\left(x_{+}\right), \quad \bar{\psi}_{2}=\bar{\psi}_{2}\left(x_{-}\right) .
\end{gathered}
$$

Using $\left[x_{+}, x_{-}\right]=2 \theta$, the expression $\psi_{\beta}(x) \star \bar{\psi}_{\alpha}(x)\left(\gamma_{\mu}\right)^{\alpha \beta}$ with on-shell Dirac fermions is rewritten as

$$
\begin{aligned}
\psi_{\beta} \star \bar{\psi}_{\alpha}\left(\gamma_{+}\right)_{\alpha \beta} & =-2 i \psi_{2}\left(x_{+}\right) \star \bar{\psi}_{1}\left(x_{+}\right) \\
& =-2 i \psi_{2}\left(x_{+}\right) \bar{\psi}_{1}\left(x_{+}\right), \\
\psi_{\beta} \star \bar{\psi}_{\alpha}\left(\gamma_{-}\right)_{\alpha \beta} & =+2 i \psi_{1}\left(x_{-}\right) \star \bar{\psi}_{2}\left(x_{-}\right) \\
& =+2 i \psi_{1}\left(x_{-}\right) \bar{\psi}_{2}\left(x_{-}\right) .
\end{aligned}
$$

With Eq. (2.30), it would be possible to find all the components of $\mathcal{Q}_{\mu \nu}$. Since there is no singularity in $\mathcal{Q}_{\mu \nu}(x, \epsilon)$, we have

$$
\lim _{\epsilon \rightarrow 0} \mathcal{Q}_{\mu \nu}(x, \epsilon)=\mathcal{Q}_{\mu \nu}(x),
$$

and hence

$$
\begin{aligned}
\mathcal{Q}_{\mu \nu}(x)= & :\left(\psi_{\beta}(x) \star \bar{\psi}_{\alpha}(x)\right) \star\left(\psi_{\sigma}(x) \star \bar{\psi}_{\rho}(x)\right): \\
& \times\left(\gamma_{\mu}\right)^{\alpha \beta}\left(\gamma_{\nu}\right)^{\rho \sigma} \\
& +:\left(\psi_{\beta}(x) \star \bar{\psi}_{\alpha}(x)\right) \star\left(\psi_{\sigma}(x) \star \bar{\psi}_{\rho}(x)\right): \\
& \times\left(\gamma_{\nu}\right)^{\alpha \beta}\left(\gamma_{\mu}\right)^{\rho \sigma} \\
& -:\left(\psi_{\beta}(x) \star \bar{\psi}_{\alpha}(x)\right) \star\left(\psi_{\sigma}(x) \star \bar{\psi}_{\rho}(x)\right): \delta_{\mu \nu} \\
& \times\left(\gamma_{\lambda}\right)^{\alpha \beta}\left(\gamma^{\lambda}\right)^{\rho \sigma} .
\end{aligned}
$$

One may then readily show that

$$
\begin{aligned}
\mathcal{Q}_{++} & =-8:\left(\psi_{2}\left(x_{+}\right) \bar{\psi}_{1}\left(x_{+}\right)\right) \star\left(\psi_{2}\left(x_{+}\right) \bar{\psi}_{1}\left(x_{+}\right)\right): \\
& =-8: \psi_{2}\left(x_{+}\right) \bar{\psi}_{1}\left(x_{+}\right) \psi_{2}\left(x_{+}\right) \bar{\psi}_{1}\left(x_{+}\right): .
\end{aligned}
$$


Performing some straightforward permutations, we get $\mathcal{Q}_{++}$ $=0$. Similarly

$$
\begin{aligned}
\mathcal{Q}_{--} & =-8:\left(\psi_{1}\left(x_{-}\right) \bar{\psi}_{2}\left(x_{-}\right)\right) \star\left(\psi_{1}\left(x_{-}\right) \bar{\psi}_{2}\left(x_{-}\right)\right): \\
& =-8: \psi_{1}\left(x_{-}\right) \bar{\psi}_{2}\left(x_{-}\right) \psi_{1}\left(x_{-}\right) \bar{\psi}_{2}\left(x_{-}\right): \\
& =0 .
\end{aligned}
$$

Also for off-diagonal components $\mathcal{Q}_{ \pm \mp}$, we have

$$
\begin{aligned}
\mathcal{Q}_{ \pm \mp}(x)= & :\left(\psi_{\beta}(x) \star \bar{\psi}_{\alpha}(x)\right) \star\left(\psi_{\sigma}(x) \star \bar{\psi}_{\rho}(x)\right): \\
& \times\left(\gamma_{ \pm}\right)^{\alpha \beta}\left(\gamma_{\mp}\right)^{\rho \sigma} \\
& +:\left(\psi_{\beta}(x) \star \bar{\psi}_{\alpha}(x)\right) \star\left(\psi_{\sigma}(x) \star \bar{\psi}_{\rho}(x)\right): \\
& \times\left(\gamma_{\mp}\right)^{\alpha \beta}\left(\gamma_{ \pm}\right)^{\rho \sigma} \\
& -: 2\left(\psi_{\beta}(x) \star \bar{\psi}_{\alpha}(x)\right) \star\left(\psi_{\sigma}(x) \star \bar{\psi}_{\rho}(x)\right): \\
& \times\left(\gamma_{\lambda}\right)^{\alpha \beta}\left(\gamma^{\lambda}\right)^{\rho \sigma} .
\end{aligned}
$$

Inserting $\gamma^{ \pm}=\frac{1}{2} \gamma_{\mp}$ results in

$$
\begin{aligned}
\mathcal{Q}_{ \pm \mp}(x)= & :\left(\psi_{\beta}(x) \star \bar{\psi}_{\alpha}(x)\right) \star\left(\psi_{\sigma}(x) \star \bar{\psi}_{\rho}(x)\right): \\
& \times\left(\gamma_{ \pm}\right)^{\alpha \beta}\left(\gamma_{\mp}\right)^{\rho \sigma} \\
& +:\left(\psi_{\beta}(x) \star \bar{\psi}_{\alpha}(x)\right) \star\left(\psi_{\sigma}(x) \star \bar{\psi}_{\rho}(x)\right): \\
& \times\left(\gamma_{\mp}\right)^{\alpha \beta}\left(\gamma_{ \pm}\right)^{\rho \sigma} \\
& -:\left(\psi_{\beta}(x) \star \bar{\psi}_{\alpha}(x)\right) \star\left(\psi_{\sigma}(x) \star \bar{\psi}_{\rho}(x)\right): \\
& \times\left(\gamma_{ \pm}\right)^{\alpha \beta}\left(\gamma_{\mp}\right)^{\rho \sigma} \\
& -:\left(\psi_{\beta}(x) \star \bar{\psi}_{\alpha}(x)\right) \star\left(\psi_{\sigma}(x) \star \bar{\psi}_{\rho}(x)\right): \\
& \times\left(\gamma_{\mp}\right)^{\alpha \beta}\left(\gamma_{ \pm}\right)^{\rho \sigma} \\
= & 0 .
\end{aligned}
$$

Consequently $Q_{\mu \nu}=0$. This means that the equivalence of the Sugawara energy-momentum tensor and energymomentum tensor $T_{\mu \nu}^{s^{\star}}=T_{\mu \nu}^{\star}$ in two-dimensional noncommutative space for free massless fermions is still satisfied. Also this equivalence occurs for the Sugawara form in terms of the current $\mathcal{J}_{\mu}(x)$, as defined in (2.7). We have

$$
\begin{aligned}
\widehat{T}_{\mu \nu}^{s^{\star}}= & \frac{\pi}{2}\left(\mathcal{J}_{\mu}(x) \star \mathcal{J}_{\nu}(x)+\mathcal{J}_{\nu}(x) \star \mathcal{J}_{\mu}(x)\right. \\
& \left.-\delta_{\mu \nu} \mathcal{J}^{\lambda}(x) \star \mathcal{J}_{\lambda}(x)\right) .
\end{aligned}
$$

To show this, let us write first all the components of the currents $J_{\mu}(x)$ and $\mathcal{J}_{\mu}(x)$ using the representation of the gamma matrices (2.26) as follows:

$$
\begin{aligned}
J_{1}(x) & =i:\left(\psi_{1}\left(x_{-}\right) \star \bar{\psi}_{2}\left(x_{-}\right)-\psi_{2}\left(x_{+}\right) \star \bar{\psi}_{1}\left(x_{+}\right)\right): \\
& =i:\left(\psi_{1}\left(x_{-}\right) \bar{\psi}_{2}\left(x_{-}\right)-\psi_{2}\left(x_{+}\right) \bar{\psi}_{1}\left(x_{+}\right)\right): \\
J_{2}(x) & =-:\left(\psi_{2}\left(x_{+}\right) \star \bar{\psi}_{1}\left(x_{+}\right)+\psi_{1}\left(x_{-}\right) \star \bar{\psi}_{2}\left(x_{-}\right)\right): \\
& =-:\left(\psi_{2}\left(x_{+}\right) \bar{\psi}_{1}\left(x_{+}\right)+\psi_{1}\left(x_{-}\right) \bar{\psi}_{2}\left(x_{-}\right)\right):
\end{aligned}
$$

as well as

$$
\begin{aligned}
\mathcal{J}_{1}(x) & =i:\left(\bar{\psi}_{2}\left(x_{-}\right) \star \psi_{1}\left(x_{-}\right)-\bar{\psi}_{1}\left(x_{+}\right) \star \psi_{2}\left(x_{+}\right)\right): \\
& =i:\left(\bar{\psi}_{2}\left(x_{-}\right) \psi_{1}\left(x_{-}\right)-\bar{\psi}_{1}\left(x_{+}\right) \psi_{2}\left(x_{+}\right)\right): \\
\mathcal{J}_{2}(x) & =-:\left(\bar{\psi}_{1}\left(x_{+}\right) \star \psi_{2}\left(x_{+}\right)+\bar{\psi}_{2}\left(x_{-}\right) \star \psi_{1}\left(x_{-}\right)\right): \\
& =-:\left(\bar{\psi}_{1}\left(x_{+}\right) \psi_{2}\left(x_{+}\right)+\bar{\psi}_{2}\left(x_{-}\right) \psi_{1}\left(x_{-}\right)\right): .
\end{aligned}
$$

We notice that the star-product appearing in the noncommutative currents is removed. Applying the permutation on the fermionic fields of the relation (2.38), we obtain

$\mathcal{J}_{\mu}(x)=-J_{\mu}(x)$

This is an interesting result in two dimensions. Unlike the four-dimensional case, where $J_{\mu}(x)$ and $\mathcal{J}_{\mu}(x)$ correspond to each other by the charge conjugation transformation [16], which is not conserved, in two dimensions the charge conjugation, as well as the Lorentz invariance, retain the symmetry of the theory as in their commutative case. Inserting (2.40) in (2.37) then leads to

$\widehat{T}_{\mu \nu}^{s^{\star}}=T_{\mu \nu}^{s^{\star}}=T_{\mu \nu}^{\star}$.

One of the physical consequences of this equivalence is noncommutative bosonization, which is obtained by writing the transformation of the field $\psi$ under the spatial translation

$\partial_{x_{1}} \psi(x)=i\left[P_{1}, \psi(x)\right], \quad P_{1}=\int \mathrm{d} x_{1}^{\prime} T_{21}^{s^{\star}}$,

where $T_{21}^{s^{\star}}$ is the conserved current arising from translational invariance. ${ }^{2}$ We have

$\partial_{x_{1}} \psi(x)=i\left[\int \mathrm{d} x_{1}^{\prime} T_{21}^{s^{\star}}, \psi(x)\right]_{x_{2}=x_{2}^{\prime}}$,

and substituting the value of $T_{21}^{s^{\star}}$ from (2.8) in (2.43) yields

$$
\begin{aligned}
\partial_{x_{1}} \psi(x)=\frac{i \pi}{2}\left[\int \mathrm { d } x _ { 1 } ^ { \prime } \left(J_{1}\left(x^{\prime}\right) \star J_{2}\left(x^{\prime}\right)\right.\right. \\
\\
\left.\left.\quad+J_{2}\left(x^{\prime}\right) \star J_{1}\left(x^{\prime}\right)\right), \psi(x)\right]_{x_{2}=x_{2}^{\prime}} .
\end{aligned}
$$

To simplify (2.44), we insert $\int \mathrm{d} x_{2}^{\prime} \delta\left(x_{2}-x_{2}^{\prime}\right)=1$ to use the trace property of the star-product, which is given by

$$
\int \mathrm{d} x_{1}^{\prime} \mathrm{d} x_{2}^{\prime} J_{2}\left(x^{\prime}\right) \star J_{1}\left(x^{\prime}\right)=\int \mathrm{d} x_{1}^{\prime} \mathrm{d} x_{2}^{\prime} J_{1}\left(x^{\prime}\right) \star J_{2}\left(x^{\prime}\right) .
$$

\footnotetext{
${ }^{2}$ In two-dimensional Euclidean space $x_{2}=i x_{0}$.
} 
Inserting (2.45) in (2.44) and applying the operator definition of the star-product (2.11), we have

$$
\begin{aligned}
\partial_{x_{1}} \psi(x)= & i \pi \int \mathrm{d} x_{1}^{\prime} \mathrm{d} x_{2}^{\prime} \delta\left(x_{2}-x_{2}^{\prime}\right) \mathcal{F}_{a b} \\
& \times\left.\left[J_{1}\left(x^{\prime}+a\right) J_{2}\left(x^{\prime}+b\right), \psi(x)\right]\right|_{a, b=0} \\
= & i \pi \int \mathrm{d} x_{1}^{\prime} \mathrm{d} x_{2}^{\prime} \delta\left(x_{2}-x_{2}^{\prime}\right) \mathcal{F}_{a b} \\
& \times\left(J_{1}\left(x^{\prime}+a\right)\left[J_{2}\left(x^{\prime}+b\right), \psi(x)\right]\right. \\
& \left.+\left[J_{1}\left(x^{\prime}+a\right), \psi(x)\right] J_{2}\left(x^{\prime}+b\right)\right)\left.\right|_{a, b=0} .
\end{aligned}
$$

The bracket terms appearing in the right-hand side of (2.46) are derived by considering the quantization condition $\left\{\psi_{\alpha}(x), \psi_{\beta}^{\dagger}\left(x^{\prime}\right)\right\}=\delta_{\alpha \beta} \delta\left(x_{1}-x_{1}^{\prime}\right)$ as follows:

$\left[J_{2}\left(x^{\prime}+a\right), \psi(x)\right]_{x_{2}=x_{2}^{\prime}}=\psi\left(x^{\prime}+a\right) \delta\left(x_{1}-x_{1}^{\prime}+a\right)$,

$\left[J_{1}\left(x^{\prime}+a\right), \psi(x)\right]_{x_{2}=x_{2}^{\prime}}=\gamma_{5} \psi\left(x^{\prime}+a\right) \delta\left(x_{1}-x_{1}^{\prime}+a\right)$,

with $\gamma_{5}=i \gamma_{1} \gamma_{2}$. Substituting (2.47) into (2.46) and then converting the result into the star-product form, we have

$\partial_{x_{1}} \psi(x)=i \pi\left(J_{1}(x)+J_{2}(x) \gamma_{5}\right) \star \psi(x)$.

The solution of this equation is represented by

$\psi(x)=\mathcal{P}\left(e_{\star}^{i \pi \int_{-\infty}^{x_{1}} \mathrm{~d} x_{1}^{\prime}\left[J_{1}\left(x^{\prime}\right)+J_{2}\left(x^{\prime}\right) \gamma_{5}\right]}\right) \psi_{0}$,

where $\mathcal{P}$ denotes the path-ordering operator and $\psi_{0}$ is a constant spinor in two dimensions.

Now, as a result of (2.38), we can use the bosonized form of the commutative current, which is introduced in Appendix A. Hence, we conclude

$\psi(x)=\mathcal{P}\left(e_{\star}^{-i \sqrt{\pi}\left[\gamma_{5} \phi(x)-\int_{-\infty}^{x_{1}} \mathrm{~d} x_{1}^{\prime} \dot{\phi}\left(x^{\prime}\right)\right]}\right) \psi_{0}$,

where $\dot{\phi}=\partial_{x_{2}^{\prime}} \phi$.

\section{Discussion}

In this paper, we established the noncommutative extension of the Sugawara construction in the bilinear form of the currents for free massless fermions in two dimensions. It was shown that this construction is precisely equivalent to the symmetric energy-momentum tensor.

To prove the correctness of this equivalence, we determined the energy-momentum tensor in two separate meth- ods. The first was the direct calculation using the symmetric definition of the energy-momentum tensor for on-shell Dirac fermions and the second contained a detailed analysis of noncommutative Sugawara construction by applying the point-splitting regularization. Furthermore, for simplification in our calculation, we considered the light-cone system. In this coordinate, we realized that the currents $J_{\mu}$ and $\mathcal{J}_{\mu}$, apart from a minus sign, are actually the same in two dimensions, which leads to the charge conjugation symmetry restoration.

Eventually, we presented a physical consequence of this equivalence, named noncommutative bosonization (e.g. see [17-19]), which relates a fermionic field to a bosonic field through an exponential function and demonstrated that a free massless fermion theory with a global $U(1)$ symmetry in noncommutative space corresponds to a free massless boson theory. Also, the bosonized version of a theory with local $U(1)$ symmetry such as two-dimensional noncommutative QED (NC-QED $\left.{ }_{2}\right)$ was addressed in [20], where it was proven that the bosonized action contains a noncommutative WessZumino-Witten (WZW) part, a gauge kinetic part, and an interaction part between the WZW and gauge field.

The physical significance of the bosonization procedure is that it specifies a duality between the strong and weak couplings for particular interacting quantum field theories. The most famous example of this duality is the equivalence of the massive Thirring model and the sine-Gordon model [21, 22 ], where the weak coupling $\beta$ of the bosonic theory, that is, the sine-Gordon model, is related to the strong coupling $g$ of the fermionic theory, the massive Thirring model, through the bosonization rule described by $\frac{4 \pi}{\beta^{2}}=1+\frac{g}{\pi}$. Moreover, the duality between the noncommutative version of these models was studied in [17-19] where it was shown that the strongweak duality is also preserved. However, it is notable that the strong-weak duality does not appear in the case of NC$\mathrm{QED}_{2}$ and its bosonized version, because of the appearance of the same couplings in two theories.

Acknowledgments I am grateful to M.M. Sheikh-Jabbari for numerous fruitful discussions and careful reading of the manuscript. I would also like to thank M. Khorrami for his valuable comments and I appreciate the insightful and constructive remarks of M. Chaichian and the referee, which led to improvement of the manuscript. Moreover, I acknowledge the School of Physics of Institute for research in fundamental sciences (IPM) for the research facilities and environment.

Open Access This article is distributed under the terms of the Creative Commons Attribution 4.0 International License (http://creativecomm ons.org/licenses/by/4.0/), which permits unrestricted use, distribution, and reproduction in any medium, provided you give appropriate credit to the original author(s) and the source, provide a link to the Creative Commons license, and indicate if changes were made.

Funded by SCOAP ${ }^{3}$. 


\section{Appendix A: Commutative Sugawara construction}

In this appendix, we present a detailed analysis on the proof of the relation $T_{\mu \nu}^{s}=T_{\mu \nu}$ in two-dimensional commutative space for free massless fermions, as argued in [5], and describe some interesting consequences of this equivalence [23-27]. The Lagrangian for the massless fermions is given by

$\mathcal{L}=\frac{i}{2}\left(\bar{\psi} \gamma^{\mu} \partial_{\mu} \psi-\partial_{\mu} \bar{\psi} \gamma^{\mu} \psi\right)$,

which is invariant under the global phase transformation $\psi \rightarrow e^{i \alpha} \psi$ and $\bar{\psi} \rightarrow e^{-i \alpha} \bar{\psi}$, which gives the conserved current

$j_{\mu}(x)=: \bar{\psi}(x) \gamma_{\mu} \psi(x):$.

For this theory, the symmetric energy-momentum tensor is written as follows:

$T_{\mu \nu}=\frac{i}{4}:\left(\bar{\psi} \gamma_{\mu} \partial_{\nu} \psi+\bar{\psi} \gamma_{\nu} \partial_{\mu} \psi-\partial_{\mu} \bar{\psi} \gamma_{\nu} \psi-\partial_{\nu} \bar{\psi} \gamma_{\mu} \psi\right):$

The energy-momentum tensor in Sugawara form is described by a bilinear function of the currents as

$$
T_{\mu \nu}^{s}=\frac{\pi}{2}\left(j_{\mu}(x) j_{\nu}(x)+j_{\nu}(x) j_{\mu}(x)-g_{\mu \nu} j^{\lambda}(x) j_{\lambda}(x)\right) .
$$

To show $T_{\mu \nu}^{s}=T_{\mu \nu}$, we start with (A.4) and replace $j_{\mu}(x) j_{v}(x)$ with

$$
\lim _{\epsilon \rightarrow 0}\left(j_{\mu}(x+\epsilon) j_{v}(x)-\left\langle j_{\mu}(x+\epsilon) j_{v}(x)\right\rangle\right) .
$$

Applying Wick's theorem to (A.5)

$$
\begin{aligned}
j_{\mu}(x+\epsilon) j_{\nu}(x)= & : \bar{\psi}(x+\epsilon) \gamma_{\mu} \psi(x+\epsilon):: \bar{\psi}(x) \gamma_{\nu} \psi(x): \\
= & : \bar{\psi}(x+\epsilon) \gamma_{\mu} \psi(x+\epsilon) \bar{\psi}(x) \gamma_{\nu} \psi(x): \\
& +: \bar{\psi}(x+\epsilon) \gamma_{\mu}\langle\psi(x+\epsilon) \bar{\psi}(x)\rangle \gamma_{\nu} \psi(x): \\
& +: \bar{\psi}(x) \gamma_{\nu}\langle\psi(x) \bar{\psi}(x+\epsilon)\rangle \gamma_{\mu} \psi(x+\epsilon): \\
& -\operatorname{tr}\left(\gamma_{\mu}\langle\psi(x) \bar{\psi}(x+\epsilon)\rangle\right. \\
& \left.\times \gamma_{\nu}\langle\psi(x+\epsilon) \bar{\psi}(x)\rangle\right),
\end{aligned}
$$

and implementing a similar analysis for the other terms of (A.4), we arrive at

$$
\begin{aligned}
T_{\mu \nu}^{s}= & \frac{\pi}{2} \lim _{\epsilon \rightarrow 0}\left[\mathcal{M}_{\mu \nu}(x, \epsilon)+\mathcal{N}_{\mu \nu}(x, \epsilon)+\mathcal{N}_{\nu \mu}(x, \epsilon)\right. \\
& +\mathcal{N}_{\mu \nu}(x,-\epsilon)+\mathcal{N}_{\nu \mu}(x,-\epsilon) \\
& \left.-g_{\mu \nu}\left[\mathcal{N}_{\lambda}^{\lambda}(x, \epsilon)+\mathcal{N}_{\lambda}^{\lambda}(x,-\epsilon)\right]\right]
\end{aligned}
$$

where $\mathcal{M}_{\mu \nu}$ and $\mathcal{N}_{\mu \nu}$ are defined as

$$
\begin{aligned}
\mathcal{M}_{\mu \nu}(x, \epsilon)= & : \bar{\psi}(x+\epsilon) \gamma_{\mu} \psi(x+\epsilon) \bar{\psi}(x) \gamma_{\nu} \psi(x): \\
& +: \bar{\psi}(x+\epsilon) \gamma_{\nu} \psi(x+\epsilon) \bar{\psi}(x) \gamma_{\mu} \psi(x): \\
& -: \bar{\psi}(x+\epsilon) \gamma_{\lambda} \psi(x+\epsilon) \bar{\psi}(x) \gamma^{\lambda} \psi(x): g_{\mu \nu}, \\
\mathcal{N}_{\mu \nu}(x, \epsilon)= & : \bar{\psi}(x+\epsilon) \gamma_{\mu} S^{(+)}(\epsilon) \gamma_{\nu} \psi(x):,
\end{aligned}
$$

and we have

$S^{(+)}(\epsilon)=\langle\psi(x+\epsilon) \bar{\psi}(x)\rangle=-\left(\frac{i}{2 \pi}\right) \frac{\epsilon_{\alpha} \gamma^{\alpha}}{\epsilon^{2}}$.

First, we concentrate on determining the value of $\mathcal{M}_{\mu v}(x)$ :

$$
\begin{gathered}
\mathcal{M}_{00}=\mathcal{M}_{11}=:\left(j_{0}\right)^{2}+\left(j_{1}\right)^{2}:, \\
\mathcal{M}_{01}=\mathcal{M}_{10}=: j_{0} j_{1}+j_{1} j_{0}:
\end{gathered}
$$

Choosing $\gamma_{0}=\sigma_{z}$ and $\gamma_{1}=i \sigma_{y}$, we find

$j_{0}=\bar{\psi}_{1} \psi_{1}-\bar{\psi}_{2} \psi_{2}, \quad j_{1}=\bar{\psi}_{1} \psi_{2}-\bar{\psi}_{2} \psi_{1}$.

Therefore, all the components of $\mathcal{M}_{\mu \nu}$ in terms of the fermionic fields are given by

$$
\begin{aligned}
\mathcal{M}_{00}= & \mathcal{M}_{11}=:\left(\bar{\psi}_{1} \psi_{1}-\bar{\psi}_{2} \psi_{2}\right)^{2}+\left(\bar{\psi}_{1} \psi_{2}-\bar{\psi}_{2} \psi_{1}\right)^{2}: \\
\mathcal{M}_{01}= & \mathcal{M}_{10}=:\left(\bar{\psi}_{1} \psi_{1}-\bar{\psi}_{2} \psi_{2}\right)\left(\bar{\psi}_{1} \psi_{2}-\bar{\psi}_{2} \psi_{1}\right) \\
& +\left(\bar{\psi}_{1} \psi_{2}-\bar{\psi}_{2} \psi_{1}\right)\left(\bar{\psi}_{1} \psi_{1}-\bar{\psi}_{2} \psi_{2}\right):
\end{aligned}
$$

Performing some permutations on the fermionic fields yields $\mathcal{M}_{\mu \nu}=0$. In the next step, our purpose is to obtain the value of $\mathcal{N}_{\mu \nu}$. To this end, let us start with an expansion of the fermionic fields up to the first order in $\epsilon$

$$
\begin{aligned}
\mathcal{N}_{\mu \nu}(x, \epsilon)= & -\frac{i \epsilon^{\xi}}{2 \pi \epsilon^{2}}:\left[\bar{\psi}(x)+\epsilon^{\alpha} \partial_{\alpha} \bar{\psi}(x)\right. \\
& \left.+\mathcal{O}\left(\epsilon^{2}\right)\right] \gamma_{\mu} \gamma_{\xi} \gamma_{\nu} \psi(x): .
\end{aligned}
$$

Taking the symmetric limits (2.21),

$$
\lim _{\epsilon \rightarrow 0} \mathcal{N}_{\mu \nu}(x, \epsilon)=\frac{i}{4 \pi}: \partial^{\xi} \bar{\psi}(x) \gamma_{\mu} \gamma_{\xi} \gamma_{\nu} \psi(x):,
$$

putting (A.14) in (A.7), and using the identity (2.24) for onshell fermions in two dimensions, we find

$T_{\mu \nu}^{s}=\frac{i}{4}:\left(\bar{\psi} \gamma_{\mu} \partial_{\nu} \psi+\bar{\psi} \gamma_{\nu} \partial_{\mu} \psi-\partial_{\mu} \bar{\psi} \gamma_{\nu} \psi-\partial_{\nu} \bar{\psi} \gamma_{\mu} \psi\right):$

which is exactly equal to $T_{\mu \nu}$ as mentioned in (A.3). This equivalence suggests the existence of a canonical massless pseudo scalar field, satisfying $[\phi(x, t), \dot{\phi}(y, t)]=i \delta(x-y)$, which is related to the conserved current $j_{\mu}(x)$ through the following equation $[24,25]$ :

$j_{\mu}(x)=\frac{1}{\sqrt{\pi}} \epsilon_{\mu \nu} \partial^{\nu} \phi(x)$. 
If we substitute (A.16) into the Sugawara form (A.4) and use the identity $\epsilon_{\mu \alpha} \epsilon_{\nu \beta}=g_{\mu \beta} g_{\alpha \nu}-g_{\mu \nu} g_{\alpha \beta}$, it is found that

$T_{\mu \nu}^{s}=\frac{1}{2}\left(\partial_{\mu} \phi \partial_{\nu} \phi+\partial_{\nu} \phi \partial_{\mu} \phi-g_{\mu \nu} \partial_{\lambda} \phi \partial^{\lambda} \phi\right)$,

which describes the energy-momentum tensor for a free massless boson. Another interesting result of the Sugawara construction is that it is possible to find explicitly the fermionic field in terms of the bosonic field, as mentioned in the introduction part. To show this, we consider the equation describing the transformation of the field $\psi$ under the spatial translation [26,27],

$\partial_{x_{1}} \psi(x)=i\left[\int \mathrm{d} x^{\prime} T_{01}^{s}, \psi(x)\right], \quad P_{0}=\int \mathrm{d} x_{1}^{\prime} T_{01}^{s}$,

where $T_{01}^{S}$ is the Noether current of translational symmetry. Inserting $T_{01}^{S}$ from (A.4) into (A.18), we have

$$
\begin{aligned}
& \partial_{x_{1}} \psi(x)=\frac{i \pi}{2} \\
& \times\left[\int \mathrm{d} x_{1}^{\prime}\left(j_{0}\left(x^{\prime}\right) j_{1}\left(x^{\prime}\right)+j_{1}\left(x^{\prime}\right) j_{0}\left(x^{\prime}\right)\right), \psi(x)\right]_{x_{0}=x_{0}^{\prime}} .
\end{aligned}
$$

Applying the equal-time commutation relations

$$
\begin{aligned}
& {\left[j_{0}\left(x^{\prime}\right), \psi(x)\right]_{x_{0}=x_{0}^{\prime}}=-\psi(x) \delta\left(x_{1}-x_{1}^{\prime}\right),} \\
& {\left[j_{1}\left(x^{\prime}\right), \psi(x)\right]_{x_{0}=x_{0}^{\prime}}=-\gamma_{5} \psi(x) \delta\left(x_{1}-x_{1}^{\prime}\right),}
\end{aligned}
$$

with $\gamma_{5}=\gamma_{0} \gamma_{1}$, we arrive at

$\partial_{x_{1}} \psi(x)=-i \pi\left[j_{1}(x)+j_{0} \gamma_{5}(x)\right] \psi(x)$.

Solving this equation yields

$\psi(x)=e^{-i \pi \int_{-\infty}^{x_{1}} \mathrm{~d} x^{\prime}\left(j_{1}\left(x^{\prime}\right)+j_{0}\left(x^{\prime}\right) \gamma_{5}\right)} \psi_{0}$,

where $\psi_{0}$ is a constant spinor in space-time. In the final step, we put the bosonized form of the currents from (A.16) in (A.22),

$\psi(x)=e^{i \sqrt{\pi}\left[\gamma_{5} \phi(x)+\int_{-\infty}^{x_{1}} \mathrm{~d} x_{1}^{\prime} \dot{\phi}\left(x^{\prime}\right)\right]} \psi_{0}$.

As we see, the spinor field $\psi$ is mapped to the bosonic field $\phi$.

\section{References}

1. P. Jordan, E.P. Wigner, About the Pauli exclusion principle. Z. Phys. 47, 631 (1928)

2. M. Gell-Mann, Symmetries of baryons and mesons. Phys. Rev. 125, 1067 (1962)
3. R.F. Dashen, D.H. Sharp, Currents as coordinates for hadrons. Phys. Rev. 165, 1857 (1968)

4. H. Sugawara, A field theory of currents. Phys. Rev. 170, 1659 (1968)

5. S. Coleman, D. Gross, R. Jackiw, Fermion avatars of the Sugawara model. Phys. Rev. 180, 1359 (1969)

6. P.C.W. Davies, Equivalence of massless boson and fermion theories in curved two-dimensional space-time: Sugawara stress tensor. J. Phys. A 11, 179 (1978)

7. A. Connes, M.R. Douglas, A.S. Schwarz, Noncommutative geometry and matrix theory: compactification on tori. JHEP 9802, 003 (1998). arXiv:hep-th/9711162

8. F. Ardalan, H. Arfaei, M.M. Sheikh-Jabbari, Noncommutative geometry from strings and branes. JHEP 9902, 016 (1999). arXiv:hep-th/9810072

9. M.R. Douglas, N.A. Nekrasov, Noncommutative field theory. Rev. Mod. Phys. 73, 977 (2001). arXiv:hep-th/0106048

10. R. Banerjee, C.K. Lee, H.S. Yang, Seiberg-Witten-type maps for currents and energy momentum tensors in noncommutative gauge theories. Phys. Rev. D 70, 065015 (2004). arXiv:hep-th/0312103

11. A. Das, J. Frenkel, On the energy-momentum tensor in noncommutative gauge theories. Phys. Rev. D 67, 067701 (2003). arXiv:hep-th/0212122

12. M. Hayakawa, Perturbative analysis on infrared aspects of noncommutative QED on R**4. Phys. Lett. B 478, 394 (2000). arXiv:hep-th/9912094

13. M. Hayakawa, Perturbative analysis on infrared and ultraviolet aspects of noncommutative QED on $\mathrm{R} * * 4$ (1999). arXiv:hep-th/9912167

14. A.M. Ghezelbash, S. Parvizi, Gauged noncommutative WessZumino-Witten models. Nucl. Phys. B 592, 408 (2001). arXiv:hep-th/0008120

15. J.S. Schwinger, Field theory commutators. Phys. Rev. Lett. 3, 296 (1959)

16. M.M. Sheikh-Jabbari, C, P, and T invariance of noncommutative gauge theories. Phys. Rev. Lett. 84, 5265 (2000). arXiv:hep-th/0001167

17. C. Nunez, K. Olsen, R. Schiappa, From noncommutative bosonization to S duality. JHEP 0007, 030 (2000). arXiv:hep-th/0005059

18. M.T. Grisaru, L. Mazzanti, S. Penati, L. Tamassia, Some properties of the integrable noncommutative sine-Gordon system. JHEP 0404, 057 (2004). arXiv:hep-th/0310214

19. H. Blas, Bosonized noncommutative bi-fundamental fermion and S-duality. JHEP 0506, 022 (2005). arXiv:hep-th/0504140

20. F. Ardalan, M. Ghasemkhani, N. Sadooghi, On the mass spectrum of noncommutative Schwinger model in Euclidean $\mathbb{R}^{2}$ space. Eur. Phys. J. C 71, 1606 (2011). arXiv:1011.4877 [hep-th]

21. S.R. Coleman, The quantum sine-Gordon equation as the massive Thirring model. Phys. Rev. D 11, 2088 (1975)

22. S. Mandelstam, Soliton operators for the quantized sine-Gordon equation. Phys. Rev. D 11, 3026 (1975)

23. Y. Freundlich, Fermions and bosons in a two-dimensional world. Nucl. Phys. B 36, 621 (1972)

24. Y. Freundlich, D. Lurie, Sugawara model and goldstone bosons. Phys. Rev. D 1, 1660 (1970)

25. Y. Freundlich, Sugawara model, broken symmetries, and masslessboson fields, ibid. Phys. Rev. D 1, 3290 (1970)

26. C.M. Sommerfield, Currents as dynamical variables. Phys. Rev. 176, 2019 (1968)

27. J.F. Willemsen, Remarks on the physical degrees of freedom in two-dimensional electrodynamics. Phys. Rev. D 9, 3570 (1974) 